\title{
Respuesta inflamatoria a nivel intrapulmonar en enfermos críticos con neumonía
}

\section{Á. Estella}

Servicio de Medicina Intensiva, Hospital SAS de Jerez, Jerez de la Frontera, Cádiz, España

Recibido el 11 de febrero de 2010; aceptado el 27 de mayo de 2010

Disponible en Internet el 10 de agosto de 2010

\author{
PALABRAS CLAVE \\ Citoquinas; \\ Neumonía; \\ Lavado \\ broncoalveolar; \\ Ventilación mecánica
}

\begin{abstract}
Resumen
Objetivo: Evaluar la relación entre el resultado microbiológico y la concentración de citoquinas en el lavado broncoalveolar (LBA).

Diseño: Estudio prospectivo observacional.

Ámbito: Unidad de cuidados intensivos ( $\mathrm{UCl})$ medicoquirúrgica de 17 camas.

Pacientes: Enfermos consecutivos en ventilación mecánica con sospecha clínica de neumonía ingresados en $\mathrm{UCl}$ durante un periodo de 27 meses.

Intervenciones: Se realizó LBA con $150 \mathrm{ml}$ de suero salino fisiológico repartidos en 3 alicuotas de $50 \mathrm{ml}$, durante el procedimiento no se utilizaron anestésicos locales. Una muestra del LBA fue procesada para su estudio microbiológico, otra cantidad de LBA se envió a laboratorio para medición de las siguientes citoquinas: interleuquina (IL) 6, IL 8, factor de necrosis tumoral alfa (TNF- $\alpha$ ), factor estimulante de crecimiento de granulocitos (G-CSF) y factor estimulante de crecimiento de granulocitos y monocitos.

Principales variables de interés: Edad, APACHE II al ingreso, días en ventilación mecánica, estancia en $\mathrm{UCl}$, mortalidad, antibioterapia previa, resultado microbiológico y concentración de citoquinas en el LBA.

Resultados: 59 enfermos fueron incluidos, la mayoría se encontraban con antibioterapia previa (79,7\%). En el análisis microbiológico 22 pacientes (37,2\%) presentaron LBA positivo. En el grupo de enfermos con LBA positivo la concentración de TNF- $\alpha$ fue más alta de forma significativa con respecto al grupo con LBA negativo.

Conclusiones: Hay una correlación significativa entre el resultado microbiológico y la concentración de TNF- $\alpha$ en el LBA.

En enfermos en ventilación mecánica, la concentración de TNF- $\alpha$ en el LBA se ha asociado al resultado positivo del cultivo a pesar de antibioterapia previa.

(C) 2010 Elsevier España, S.L. y SEMICYUC. Todos los derechos reservados.
\end{abstract}




\section{KEYWORDS}

Cytokines;

Pneumonia;

Bronchoalveolar

lavage;

Mechanical

ventilation

\section{Intrapulmonary inflammatory response in critically ill patients with pneumonia}

\begin{abstract}
Objective: To evaluate the relationship between the microbiology result and cytokine expression in bronchoalveolar lavage (BAL).

Design: An observational, prospective study.

Setting: A 17-bed medical and surgical intensive care unit.

Patients: Mechanically-ventilated patients with suspected pneumonia admitted to the ICU during a 27-month time period were consecutively enrolled.

Interventions: BAL was performed with $150 \mathrm{ml}$ sterile isotonic saline solution in three aliquots of $50 \mathrm{ml}$. Local anesthetics were not used during the procedure. A BAL sample was processed for a microbiologic quantitative culture and BAL cytokines IL- 6, IL 8 , TNF $\alpha$, granulocyte colony-stimulating factor (G-CSF) and granulocyte-monocyte colony-stimulating factor (GM-CSF) were measured.

Main variables of interest: Age, APACHE II score within the first 24 hours of admission, time on mechanical ventilation, ICU length of stay, mortality, previous antibiotic therapy, isolated bacteria and cytokines concentration were analyzed.

Results: Fifty-nine consecutive patients were included, and most of the patients (79.7\%) had prior antibiotic therapy. Twenty-two patients $(37.2 \%)$ had a positive BAL. In the group of patients with positive BAL, the TNF $\alpha$ concentration was significantly higher in the group of patients with positive BAL than in the BAL negative group.

Conclusions: There is a significant correlation between the microbiology result and the TNF $\alpha$ concentration in the BAL fluid. In mechanically-ventilated patients, TNF $\alpha$ in BAL has been associated with positive cultures despite prior antibiotic therapy. (c) 2010 Elsevier España, S.L. and SEMICYUC. All rights reserved.
\end{abstract}

\section{Introducción}

La neumonía es la patología infecciosa más frecuente en las Unidades de Cuidados Intensivos ( $\mathrm{UCl}$ ), se asocia a una alta morbimortalidad. Debido a que los criterios clínicos y radiológicos tienen una baja especificidad en enfermos en ventilación mecánica el diagnóstico se basa en la asociación de estos y los cultivos microbiológicos.

A pesar de los numerosos estudios surgidos en la última década no hay un patrón de referencia universal para el diagnóstico de esta patología ${ }^{1}$, habiéndose descrito diferentes estrategias diagnósticas que hoy en día continúan en debate. En los últimos años se está discutiendo el papel de nuevas herramientas de ayuda en el diagnóstico como los biomarcadores, siendo los más estudiados la proteína $C$ reactiva, procalcitonina, STREM-1 y las citoquinas.

A nivel intrapulmonar la respuesta local frente a la infección está mediada por citoquinas. Son escasos los estudios que analizan la concentración de estos mediadores a nivel pulmonar en enfermos críticos en ventilación mecánica, además, los resultados derivados de los mismos son contradictorios. En el presente estudio hemos investigado la concentración de citoquinas en el lavado broncoalveolar (LBA) con el objetivo de analizar la relación entre la respuesta inflamatoria local mediada por citoquinas a nivel intrapulmonar y el resultado microbiológico en muestras de LBA.

\section{Pacientes y métodos}

Estudio prospectivo observacional realizado en una $\mathrm{UCI}$ medicoquirúrgica de 17 camas en el que se incluyó de forma consecutiva durante un periodo de 27 meses a pacientes en ventilación mecánica con sospecha clínica de neumonía. El estudio fue aprobado por el comité de ética local del hospital. Se realizó un LBA broncoscópico previa obtención del consentimiento informado escrito del familiar o representante del enfermo. La sospecha clínica se basó en los siguientes criterios: imagen de infiltrado en la radiografía de tórax y al menos 2 de los siguientes criterios: a) fiebre de más de $38,3^{\circ} \mathrm{C}$; b) secreciones traqueobronquiales purulentas, y c) leucocitosis $>12.000 / \mu \mathrm{l}$ o leucopenia $<4.000 / \mu \mathrm{l}$.

Fueron excluidos del estudio aquellos enfermos cuya situación clínica y/o hemodinámica no garantizaba la realización de la fibrobroncoscopia con seguridad: tubo endotraqueal menor de 8 milímetros de diámetro, saturación de oxígeno, determinada mediante pulsioximetría, menor de $90 \%$ con $\mathrm{FIO}_{2}$ de 1 , acidosis severa $(\mathrm{pH}<7,20)$ e inestabilidad hemodinámica definida por tensión arterial sistólica $<90 \mathrm{mmHg}$ a pesar de fármacos vasoactivos, y aquellos que en el momento del estudio estaban en tratamiento con corticoides. En cada caso se registraron los siguientes datos: edad, índice de gravedad APACHE II al ingreso en $\mathrm{UCl}$, antibioterapia previa, diagnóstico al ingreso en $\mathrm{UCl}$, días en ventilación mecánica, estancia en $\mathrm{UCl}$, mortalidad y resultado microbiológico del LBA.

Se realizó LBA con $150 \mathrm{ml}$ de suero salino fisiológico, repartidos en 3 alícuotas de $50 \mathrm{ml}$, e instilados en la localización sugerida por la radiografía de tórax o a nivel de lóbulo medio derecho en el caso de infiltrado bilateral. No se administraron anestésicos locales y se evitó la aspiración a través del canal del broncoscopio hasta enclavar el mismo en la localización a estudiar. Al realizar 
el LBA, los primeros $20 \mathrm{ml}$ recuperados fueron desechados y una muestra del líquido restante fue procesado para estudio microbiológico. El LBA se consideró positivo con crecimiento de más de $10^{4}$ unidades formadoras de colonia $/ \mathrm{ml}$ (ufc/ $\mathrm{ml}$ ) y fue considerado la prueba de referencia para el diagnóstico de neumonía microbiologicamente confirmada. En laboratorio se realizó tinción directa de la muestra (tinción de Gram), tinción para micobacterias y hongos, cultivo bacteriano en los medios habituales (agar sangre, agar chocolate, MacConkey, agar Brucella y $\mathrm{BHI}$ ) y en medios específicos para Mycobacteria (medio líquido: Bactec MGIT-960-BD), hongos (agar Sabouraud) y Legionella (BCYE). También se aplicaron técnicas de inmunofluorescencia para Legionella. No se realizó estudio citológico.

Otra cantidad del líquido del LBA, de aproximadamente $25 \mathrm{ml}$, se envió a laboratorio para medición de citoquinas mediante inmunoanálisis ligado a enzimas (ELISA) para la cuantificación in vitro de interleuquina 6 (IL-6), interleuquina 8 (IL-8), factor de necrosis tumoral $\alpha$ ( $\alpha$-TNF), factor estimulante de crecimiento de granulocitos (GCSF) y factor estimulante de crecimiento de colonias de granulocitos y monocitos (GM-CSF). Las muestras se recogieron en tubos estériles, libres de pirógenos y otras toxinas y sin sustancias anticoagulantes. Fueron centrifugadas rápidamente a $3.000 \mathrm{rpm}$ durante $10 \mathrm{~min}$, alicuotado el suero $(250-500 \mu \mathrm{l})$ para evitar ciclos de congelacióndescongelación que pueden dar lugar a modificaciones erróneas de los valores y se almacenaron $\mathrm{a}-70{ }^{\circ} \mathrm{C}$ hasta su procesamiento. La sensibilidad del test fue de 2, 25,12, 39 y $7,8 \mathrm{pg} / \mathrm{ml}$ para la IL-6, IL8, $\alpha$-TNF, G-CSF y GM-CSF respectivamente, no se han descrito actividad ni reacciones cruzadas para ninguna de las técnicas utilizadas.

Los datos recogidos fueron analizados mediante el programa estadístico SPSS versión 15.0 para Windows. Se consideró significativo un valor de $p<0,05$. Las variables cuantitativas se expresan en media y desviación estándar, y las variables cualitativas, en porcentaje. Para comparar variables cuantitativas entre 2 grupos se aplicó la prueba no paramétrica de Mann-Whitney al no poder asumir normalidad en la distribución de la muestra. Para analizar el papel de la concentración de $\alpha$ TNF en el LBA para discriminar en función del resultado microbiológico positivo y negativo se construyó una curva ROC no paramétrica.

\section{Resultados}

Un total de 59 enfermos fueron analizados durante el periodo de estudio, 5 enfermos fueron excluidos, 3 enfermos con enfermedad pulmonar obstructiva crónica en tratamiento con corticoides y 2 por presentar un tubo orotraqueal menor de $8 \mathrm{~mm}$ de diámetro. La edad media fue de $63,5 \pm 16$ años, la puntuación media de la escala APACHE Il al ingreso fue de $19,2 \pm 7,8$ puntos, la media de días en ventilación mecánica fue de $12,1 \pm 12$ días y la estancia media en $\mathrm{UCl}$ de 15,6 $\pm 13,2$ días. La mayoría de los enfermos presentaba antibioterapia previa en el momento de realizar el lavado broncoalveolar, tan solo 12 enfermos, un $20,3 \%$ no tenía tratamiento antibiótico. El diagnóstico al ingreso fue mayoritariamente por patología médica, un $61 \%$; 10 enfermos $(16,9 \%)$ ingresaron por patología neurológica, 7 pacientes $(11,9 \%)$ ingresaron para control postoperatorio y 6 $(10,2 \%)$ por traumatismos. El patrón radiológico predominante en el momento de la realización de la fibrobroncoscopia fue de un infiltrado bilateral en un $49,1 \%$, infiltrado unilateral en un $40,7 \%$ y atelectasia en un 10,2\%. La mortalidad fue de un $32,2 \%$.

El LBA fue positivo, definido como más de $10^{4} \mathrm{ufc} / \mathrm{ml}$, en 22 pacientes $(37,2 \%)$, no se observaron diferencias en las características clínicas y mortalidad al comparar los grupos según el resultado del LBA (tabla 1).

Tabla 1 Características clínicas según el resultado microbiológico del LBA. Datos presentados en media \pm desviación estándar

\begin{tabular}{llll}
\hline & LBA $<10^{4} \mathrm{ufc} / \mathrm{ml}(\mathrm{n}: 37)$ & LBA $>10^{4} \mathrm{ufc} / \mathrm{ml}(\mathrm{n}: 22)$ & Sig. estadística \\
\hline Edad (años) & $59,6 \pm 15,6$ & $58,1 \pm 14,3$ & $\mathrm{p}: \mathrm{ns}$ \\
Apache II & $19,3 \pm 8,6$ & $19 \pm 6,5$ & $\mathrm{p}: \mathrm{ns}$ \\
Días en VM & $13 \pm 13,9$ & $10,6 \pm 7,6$ & $\mathrm{p}: \mathrm{ns}$ \\
Estancia UCl & $16,8 \pm 14,9$ & $13,4 \pm 9,2$ & $\mathrm{p}: \mathrm{ns}$ \\
Mortalidad & $35,1 \%$ & $27,3 \%$ & $\mathrm{p}: \mathrm{ns}$ \\
\hline
\end{tabular}

Tabla 2 Concentración de citoquinas en el LBA $(\mathrm{pg} / \mathrm{ml})$ en función del resultado microbiológico del LBA. Datos presentados en media \pm desviación estándar

\begin{tabular}{lccl}
\hline & LBA $<10^{4} \mathrm{ufc} / \mathrm{ml}$ & LBA $>10^{4} \mathrm{ufc} / \mathrm{ml}$ & Sig. estadística \\
\hline IL6 & $180,3 \pm 252$ & $293,4 \pm 421,6$ & $\mathrm{p}: \mathrm{ns}$ \\
IL8 & $1.301,4 \pm 1.045,6$ & $1.681,1 \pm 1.315,5$ & $\mathrm{p}: \mathrm{ns}$ \\
$\alpha$ TNF & $48,9 \pm 80,7$ & $222,6 \pm 308,6$ & $\mathrm{p}: 0,02$ \\
GCSF & $444,8 \pm 565,3$ & $408,1 \pm 491,4$ & $\mathrm{p}: \mathrm{ns}$ \\
GMCSF & $14,1 \pm 23,5$ & $9,35 \pm 17,3$ & $\mathrm{p}: \mathrm{ns}$ \\
\hline
\end{tabular}




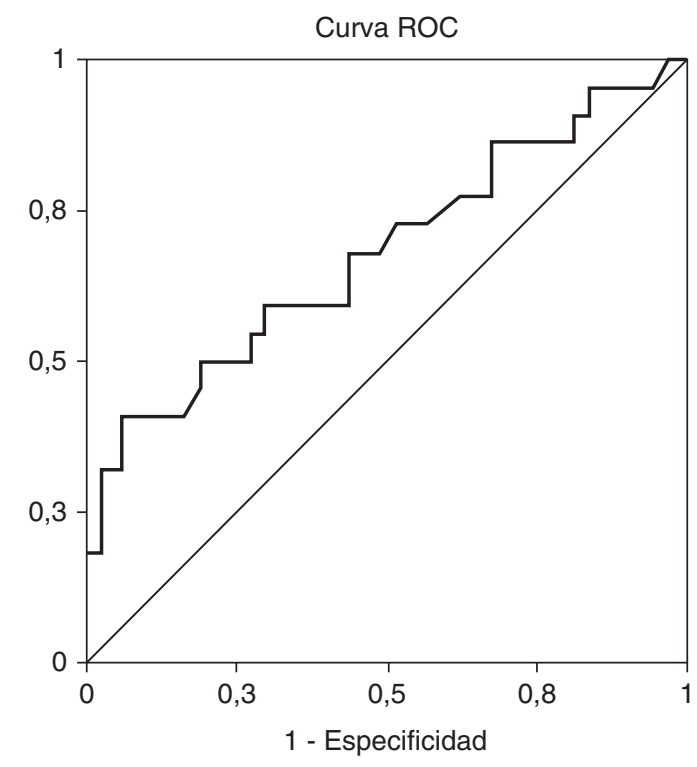

Figura 1 Curva ROC. Concentración $\alpha$-TNF en el LBA y resultado microbiológico.

En el grupo de enfermos con LBA positivo se objetivó una mayor concentración de citoquinas IL6, IL8 y $\alpha$-TNF, si bien, no hubo diferencias estadísticamente significativas salvo en la concentración de $\alpha$-TNF (tabla 2). En la curva ROC obtenida para evaluar el papel de la concentración de $\alpha$-TNF para discriminar en función del resultado del LBA (fig. 1) se obtuvo un área bajo la curva de 0,68 con un IC del 95\%: $0,53-0,83$.

\section{Discusión}

El presente estudio tiene por objeto analizar la relación entre la respuesta inflamatoria mediada por citoquinas a nivel intrapulmonar y el resultado de los cultivos microbiológicos obtenidos mediante LBA broncoscópico. Varios investigadores han determinado la concentración de citoquinas en muestras obtenidas del tracto respiratorio en enfermos críticos con patología pulmonar, si bien sus resultados han sido contradictorios, los argumentos para explicar esta circunstancia se fundamentan principalmente en las dificultades que conlleva realizar este tipo de estudios, no solo por los múltiples factores que influyen en la respuesta inflamatoria sino también por las dificultades que conlleva el diagnóstico microbiológico en este grupo de enfermos.

El diagnóstico microbiológico del enfermo crítico con sospecha de neumonía es en la actualidad, a pesar de los numerosos estudios surgidos en la última década, objeto de debate ${ }^{2,3}$, coexisten distintas estrategias diagnósticas con diferentes muestras respiratorias a utilizar, además de esta circunstancia, la principal limitación radica en que la mayoría presentan en el momento de la toma de la muestra antibioterapia previa, disminuyendo por tanto la rentabilidad de los cultivos ${ }^{4}$. En el presente estudio la mayoría de los enfermos presentaban antibioterapia previa siendo los cultivos microbiológicos positivos en un $37,2 \%$.
A esta limitación hay que añadir que en el enfermo crítico las citoquinas están implicadas en la patogenia de numerosas enfermedades ${ }^{5}$, y además, diversos factores como pueden ser el modo y tiempo en ventilación mecánica ${ }^{6,7}$, estado inmunológico, patología asociada, y microorganismos aislados, entre otros, pueden influir en dicha respuesta. En nuestra serie no hubo diferencias entre los grupos comparados respecto al estado hemodinámico, ya que un criterio de exclusión para la realización de la fibrobroncoscopia fue la existencia de inestabilidad hemodinámica.

Las infecciones a nivel pulmonar constituyen un estímulo para la activación de una respuesta inflamatoria, siendo el pulmón un órgano especialmente sensible a la producción de citoquinas. Varios autores han descrito que la respuesta inflamatoria es de carácter local en la neumonía ${ }^{8-10}$, con aumento de citoquinas intrapulmonares a nivel del pulmón afecto. Esta compartimentalización de la respuesta inflamatoria a nivel local de la infección puede desbordarse con extensión y liberación de citoquinas a la circulación sanguínea ocasionando una respuesta inflamatoria sistémica con repercusión clínica. No están bien precisados los factores que influyen en la pérdida de compartimentalización de la respuesta inflamatoria a nivel pulmonar.

De igual forma, lesiones extrapulmonares con desarrollo de respuesta inflamatoria sistémica pueden activar la producción local de citoquinas en el síndrome de distrés respiratorio agudo ${ }^{11,12}$.

En el presente estudio hemos observado una relación estadísticamente significativa entre el resultado del cultivo y la concentración de $\alpha$-TNF en el LBA, siendo esta mayor en los enfermos con cultivos positivos, de igual forma, aunque sin obtener significación estadística se observó una mayor concentración de IL-6 e IL-8 en el LBA. En concordancia con estos hallazgos $\mathrm{Wu} \mathrm{CL}$ et $\mathrm{al}^{13}$ observaron una mayor concentración de estos mediadores inflamatorios, IL-6 e IL-8 en el LBA, aunque sin ser estadísticamente significativos, objetivando una asociación significativa con la concentración de interleuquina 1- $\beta$ (IL-1 $\beta)$. Hay que destacar que al igual que en nuestro estudio se demostró la asociación de dichos mediadores con el resultado del cultivo a pesar de antibioterapia previa. Dichos resultados difieren con los observados por Monton et $\mathrm{al}^{14}$ que concluyeron que la respuesta inflamatoria a nivel pulmonar era independiente del cultivo microbiológico en enfermos críticos, argumentando que probablemente era debido a que estaban previamente tratados con antibióticos, si bien el tamaño muestral de dicho estudio fue de 30 enfermos, tan solo 20 de ellos con neumonía y, aunque sin obtener diferencias significativas también observaron una mayor concentración de estos mediadores en el LBA. Hay pocos estudios que analicen los niveles de G-CSF y GM-CSF a nivel pulmonar en enfermos $\operatorname{críticos}^{15}$, no es bien conocido el papel de estas citoquinas en la respuesta inflamatoria, en nuestra serie no se observó relación entre los niveles de estos mediadores y el resultado microbiológico.

El factor de necrosis tumoral es un glucopéptido de 185 aminoácidos, localizado genéticamente en el cromosoma $7(7 p 21)$, su producción es estimulada en respuesta a la agresión celular y a otros mediadores, como endotoxinas bacterianas y la interleuquina 1. Participa en la respuesta inflamatoria estimulando la síntesis de proteínas de fase aguda. Varios estudios han demostrado el relevante papel 
clave del $\alpha$-TNF como mediador inflamatorio ante la agresión pulmonar que conllevan infecciones respiratorias causadas por cocos Gram positivos, Legionella sp., bacilos Gram negativos y otros microorganismos ${ }^{16-18}$. En nuestro estudio el LBA fue realizado en cuanto se tuvo sospecha clínica de neumonía, en su fase inicial, $\alpha$-TNF está descrita como la primera citoquina en aparecer en estudios experimentales seguida de la IL-1 $\beta$ y la IL- $6^{19}$. El papel de esta citoquina como mediador inflamatorio que aparece en las fases precoces de la neumonía ha sido descrito tanto en pacientes como en estudios experimentales ${ }^{20-22}$.

La falta de un patrón de referencia universal para el diagnóstico de neumonía en enfermos críticos ha conllevado el auge en los últimos años de determinados biomarcadores como herramienta de ayuda ${ }^{23}$, en el presente estudio hemos observado la asociación de $\alpha$-TNF a nivel intrapulmonar, como marcador asociado al resultado positivo de los cultivos microbiológicos. En general, en las enfermedades infecciosas en el enfermo crítico el papel de los biomarcadores por si solos está limitado por su cuestionada sensibilidad, especificidad y aplicabilidad en la clínica ${ }^{24}$. Esta serie es, en el momento actual, la más amplia en número de enfermos críticos que investiga la respuesta inflamatoria a nivel pulmonar determinando las cinco citoquinas analizadas. Dentro de las limitaciones del presente estudio cabe destacar la falta de homogeneidad de los pacientes incluidos ya que las diferencias en el motivo de ingreso, tratamientos, días en ventilación mecánica, y modos de ventilación pueden influir en la respuesta inflamatoria, respecto a este punto, diferentes modos de ventilación mecánica pueden influir en la liberación de mediadores proinflamatorios ${ }^{25,26}$, la mayoría de los enfermos estudiados por nosotros fueron ventilados de la misma forma aunque no se siguió un protocolo definido específicamente para el estudio. Finalmente, solo se analizaron dichos marcadores en un solo tiempo, ante la sospecha clínica de neumonía, en la fase precoz, sin compararse con estadios más avanzados ya que, desde el punto de vista ético, no estaba justificado repetir las broncoscopias en otros tiempos con dicho fin.

\section{Bibliografía}

1. Olaechea P. Infecciones bacterianas en el paciente crítico: revisión de los estudios publicados entre 2006 y 2008. Med Intensiva. 2009;33:196-206.

2. Fagon JY, Chastre J, Wolff M, Gervais C, Parer-Aubas S, Stephan F, et al. Invasive and noninvasive strategies for management of suspected ventilator-associated pneumonia. A randomized trial. Ann Intern Med. 2000;132:621-30.

3. The Canadian Critical Care Trial Group. A randomized trial of diagnostic techniques for ventilator associated pneumonia. $N$ Engl J Med. 2006;355:2619-30.

4. Fàbregas N, Torres A, El-Ebiary $M$, Ramírez J, Hernández C, González J, et al. Histopathologic and microbiologic aspects of ventilator associated pneumonia. Anesthesiology. 1996;84:760-71.

5. Charo IF, Ransohoff $M$. The many roles of chemokines and chemokine receptors in inflammation. $N$ Engl J Med. 2006;354:610-21.

6. Stuber F, Wrigge H, Schroeder S, Wetegrove S, Zinserling J, Hoeft A, et al. Kinetic and reversibility of mechanical ventilation-associated pulmonary and systemic inflammatory response in patients with acute lung injury. Intensive Care Med. 2002;28:834-41.
7. Ranieri VM, Suter PM, Tortorella C, De Tullio R, Dayer JM, Brienza A, et al. Effect of mechanical ventilation on inflammatory mediators in patients with acute respiratory distress syndrome: a randomized controlled trial. JAMA. 1999;282:54-61.

8. Millo JL, Schultz MJ, Williams C, Weverling GJ, Ringrose T, Mackinlay $\mathrm{Cl}$, et al. Compartmentalisation of cytokines and cytokine inhibitors in ventilator-associated pneumonia. Intensive Care Med. 2004;30:68-74.

9. Dehoux MS, Boutten A, Ostinelli J, Seta M. Compartmentalized cytokine production within the human lung in unilateral pneumonia. Am J Respir Crit Care Med. 1994;150:710-6.

10. Monton C, Torres A. Lung inflammatory response in pneumonia. Monaldi Arch Chest Dis. 1998;53:56-63.

11. Schutte H, Lohmeyer J, Rosseau S, Ziegler S, Siebert C, Kielisch H, et al. Bronchoalveolar and systemic cytokine profiles in patients with ARDS, severe pneumonia and cardiogenic pulmonary oedema. Eur Respir J. 1996;9:1858-67.

12. Puneet $P$, Moochhala S, Bhatia M. Chemokines in acute respiratory distress syndrome. Am J Physiol Lung Cell Mol Physiol. 2005;288:3-15.

13. Wu CL, Lee YL, Chang KM, Chang GC, King SL, Chiang CD, et al. Bronchoalveolar interleukin-1 beta: a marker of bacterial burden in mechanicallyventilated patients with communityacquired pneumonia. Crit Care Med. 2003;31:812-7.

14. Monton C, Torres A, El-Ebiary M, Filella X, Xaubet A, Puig de la Bellacsa J. Cytokine expression in severe pneumonia: a bronchoalveolar lavage study. Crit Care Med. 1999;27: 1745-53.

15. Aggarwal A, Baker CS, Evans TW, Haslam PL. G-CSF and IL-8 but not GM-CSF correlate with severity of pulmonary neutrophilia in acute respiratory distress syndrome. Eur Respir J. 2000;15: 895-901.

16. Nelson S, Mason CM, Kolls J, Summer WR. Pathophysiology of pneumonia. Clin Chest Med. 1995;1:1-12.

17. Nelson S, Noel P, Bokulic R, Bagby G, Summer WR. Murine recombinant tumor necrosis factor enhances pulmonary host defense against Staphylococcus aureus. Am Rev Respir Dis. 1989;139:A357.

18. Maus U, Rosseau S, Knies U, Seeger W, Lohmeyer J. Expression of pro-inflammatory cytokines by flow-sorted macrophages in severe pneumonia. Eur Respir J. 1998;11:534-41.

19. Puren AJ, Feldman C, Savage N, Becker PJ, Smith C. Patterns of cytokine expression in community-acquired pneumonia. Chest. 1995;107:1342-9.

20. Laichalk LL, Kunkel SL, Strieter RM, Danforth JM, Bailie MB, Standiford TJ. Tumor necrosis factor mediates lung antibacterial host defense in murine Klebsiella pneumonia. Infect Immun. 1996;64:5211-8.

21. Brieland JK, Remick DG, Freeman PT, Hurley MC, Fantone JC, Engleberg NC. In vivo regulation of replicative Legionella pneumophila lung infection by endogenous tumor necrosis factor alpha and nitric oxide. Infect Immun. 1995;63:3253-8.

22. Mehrad B, Strieter RM, Standiford TJ. Role of TNF-alpha in pulmonary host defense in murine invasive aspergillosis. J Immunol. 1999;162:1633-40.

23. Summah $H, Q u$ Jie-Ming A. Biomarkers: A definite plus in pneumonia. Mediators Inflamm. 2009;2009:675753.

24. Pierrakos C, Vincent JL. Sepsis biomarkers: A review. Crit Care. 2010;14:R15.

25. Cheng K, Zhang $\mathrm{H}$, Lin CY, Slutsky AS. Ventilation with negative airway pressure induces a cytokine response in isolated mouse lung. Anesth Analg. 2002;94:1577-82.

26. Wilson MR, Choudhury S, Takata M. Pulmonary inflammation induced by high-stretch ventilation is mediated by tumor necrosis factor signaling in mice. Am J Physiol Lung Cell Mol Physiol. 2005;288:599-607. 